\section{ACERCA DE LA INTERCULTURALIDAD}

\section{[ABOUT INTERCULTURALITY]}

\author{
Fernando Ríos-Correa
}

Sr. Editor. He leído con mucho interés el artículo del Dr. Oswaldo Salaverry publicado en vuestra revista, titulado "Interculturalidad en la salud" (1), situación que me ha producido una gran satisfacción, pues se evidencia que un médico -considerando que la percepción general sobre la formación médica suele enmarcarse en los límites de lo científico- puede tener un enfoque amplio en cuanto a multiculturalidad, multiculturalismo e interculturalidad, términos constantes y arduamente debatidos en el campo de las humanidades y las ciencias sociales ${ }^{(2)}$.

No obstante, en algunos de los párrafos del citado manuscrito puede percibirse un aire idealista en la narración de la etapa prehispánica, donde rescata la unidad de unos pueblos, y la convivencia armónica de otros en el transcurso histórico de este lado de América, inclusive mucho antes de la existencia del mundo incaico. En ese sentido, basta combinar las narraciones de Betanzos y Garcilaso, entre otros cronistas, para notar que la fusión de nuestras culturas ancestrales, no fue un tema tan poético como el que se intentó narrar entre los años de la Reconstrucción Nacional (2) -luego de la Guerra del Pacífico- y las primeras décadas del siglo XX, de la mano de Manuel González Prada y los integrantes del Primer Movimiento Indigenista. Dentro de lo expuesto por el autor el "equilibrio" es un aspecto clave, usado, sobre todo, al describir la interacción entre una cultura y otra; precisamente el carecer de equilibrio fue un tema crucial en la llegada de los españoles a un Tahuantinsuyo infestado por guerras y desangramientos internos. No obstante, mi posición cuenta con un menor fundamento bibliográfico, en comparación a todos los autores citados en la narración acerca de las comunidades indígenas.

Tras advertir mi diferencia cordial, retorno al tema de salud y cultura, partiendo por una situación que si bien no es de muy alta frecuencia, puede fácilmente presentarse en cualquier institución hospitalaria: la discusión entre un médico a punto de entrar a sala de operaciones para salvar la vida de un niño y la madre de éste, Testigo de Jehová, que se niega a aceptar que a su vástago se le practique una transfusión sanguínea. Un caso que puede

Estudiante, Escuela de Antropología, Facultad de Ciencias Sociales, Universidad Nacional Mayor de San Marcos. Lima, Perú.

Recibido: 03-06-10 Aprobado: 09-06-10 contextualizar lo valioso del dónde, o cómo, se podría poner en evidencia la necesidad de políticas culturales insertas en el tema salud. La falta de un canal de diálogo entre ambos lados de un mismo grupo social, provoca una situación tensa y mediática, que en primera y última instancia, afecta únicamente al niño, ajeno a protocolos y evangelios. Esto concuerda con la ampliación de una visión de interculturalidad más allá de lo indígena, expuesta brillantemente por el profesor Salaverry.

La distancia que existe entre la experiencia social y el metodismo científico de la medicina, a pesar de lo descrito líneas arriba, no es irreconciliable. Por el contrario, la historia nos ha mostrado, con todas sus contradicciones, cómo es que a lo largo de los últimos cuatro siglos, la relación entre la medicina y las ciencias sociales y humanidades, pasaron de ser apasionadamente antagónicas, a permanentes cooperantes ${ }^{(3)}$. El trabajo ahora, concordando con el autor, es lograr poner en la agenda curricular de las escuelas de medicina del país lo social no como un complemento, sino como una base.

Respecto al último párrafo, debo subrayar la activa participación de muchos alumnos de las Facultades de Medicina de la Universidad Nacional Mayor de San Marcos y de la Universidad Peruana Cayetano Heredia en los movimientos estudiantiles de la década de 1980 (4), independientemente a lo pretendido, o a la visión que del tema se tenga hoy en día, el hecho de que estudiantes tan vinculados a cuestiones empíricas se hayan visto inmersos en movilizaciones que se basaban en reivindicaciones sociales, pone en evidencia que el camino para la fusión de ambas ciencias, después de todo, no será gigantescamente complicado.

Por último, debo de resaltar la disposición del Comité Editor en constituir a la Revista Peruana de Medicina Experimental y Salud Pública como un canal científico con apertura a temas sociales y de humanidades, más aun cuando la salud pública requiere de éstos elementos fundamentada en su naturaleza multidisciplinaria.

\section{Conflictos de Interés}

El autor refiere no tener conflictos de interés en la publicación de este artículo.

\section{REFERENCIAS BIBLIOGRÁFICAS}

1. Salaverry O. Interculturalidad en salud. Rev Peru Med Exp Salud Publica. 2010; 27(1): 80-93.

2. Ghezzi I. La naturaleza de la guerra prehispánica temprana: la perspectiva desde Chankillo. Revista Andina. 2007; 44: 199-225. 
3. Wallerstein I. Abrir las ciencias sociales. Informe de la Comisión Gulbenkian para la reestructuración de las Ciencias Sociales. Madrid: Siglo XXI Editores; 1996.

4. Perú, Comisión de la Verdad y Reconciliación Nacional. Las organizaciones sociales: las universidades. En: Comisión de la Verdad y Reconciliación Nacional: Informe final. Lima: CVR; 2003.

Correspondencia: Fernando Ríos Correa

Dirección: Ciudad Universitaria, Universidad Nacional Mayor de San Marcos, Pabellón de Ciencias Sociales. Av. Venezuela s/n, Lima 1, Perú.

Correo electrónico: 10150046@unmsm.edu.pe

\section{ESTUDIANTES DE PREGRADO: EL FUTURO DE LA INVESTIGACIÓN}

\section{[UNDERGRADUATE STUDENTS: THE FUTURE OF RESEARCH]}

\author{
Jorge Osada 1,3,a, Paulo Ruiz-Grosso ${ }^{1,3, a}$, \\ Mariana Ramos $1,2,3 a$
}

Sr. Editor: La investigación científica y su consiguiente publicación se han visto incrementadas en los últimos años, tanto en artículos como en el número revistas científicas que los presentan (1). Este crecimiento, influenciado por la facilidad de comunicación informática, ha sido aprovechado inmensamente por diversos grupos que se han desarrollado y afianzado sus posiciones como fuentes de conocimiento y referencia científica en diversas aéreas.

En la actualidad, se puede afirmar que navegar en la vasta red informática sin encontrar referencias científicas o a sus autores es algo inverosímil. A pesar de este desarrollo, y de su gran número de beneficios y beneficiarios, su potencial todavía no es aprovechado por todos los profesionales, científicos e investigadores. Esto va de la mano con la falta de información en los beneficios que la investigación trae tanto a nivel personal como en la sociedad.

Existe una gran cantidad de ideas de investigación no realizadas así como investigaciones truncadas y artículos no publicados. La relación entre los artículos no publicados que por ejemplo se presentan en un congreso y aquellos que terminan en una publicación puede llegar a ser hasta de 4 a $1^{(2,3)}$.

\footnotetext{
1 Facultad de Medicina, Universidad Peruana Cayetano Heredia. Lima, Perú.

2 Sociedad Científica de San Fernando, Facultad de Medicina, Universidad Nacional Mayor de San Marcos. Lima, Perú.

3 Grupo de Trabajo en Salud Mental, Universidad Peruana Cayetano Heredia. Lima, Perú.

a Médico Epidemiólogo.

Recibido: 05-03-10 Aprobado: 27-03-10
}

Nos encontramos en una situación similar a la que se vivió en épocas antiguas, tenemos un amplio mundo para descubrir y desarrollar, pero el temor a lo desconocido, es decir, a la investigación y su publicación, termina por atrapar a muchos. Lamentablemente, también retiene a aquellos que son los llamados a continuar o en muchos casos iniciar esta corriente de generación de conocimientos, nos referimos a los estudiantes universitarios.

Muchos estudiantes universitarios son expuestos a la comunidad científica de manera obligatoria y poco atractiva, siendo muchas veces presionados para realizar proyectos incluso sin haber desarrollado las capacidades mínimas para hacerlos. Así se desarrollan individuos que sólo ven a la investigación como un paso enigmático y perverso para cumplir un objetivo académico inmediato y no como una fuente válida de adquirir conocimientos y promover el desarrollo científico.

Incluso si existe evidencia que los estudiantes de medicina tienen un nivel aceptable de conocimientos en cuanto al proceso de investigación en sus instituciones $(4,5)$, esto no se ve plasmado en una mayor calidad o número de publicaciones. Para aquellos interesados en realizar investigación, surgen organizaciones mantenidas por los mismos alumnos como iniciativas extracurriculares que intentan suplir el serio abandono institucional en que se encuentra la formación en investigación ${ }^{(5)}$. Aun peor, muchas universidades han eliminado la necesidad de hacer investigación en forma de tesis como requisito para graduarse de pregrado sustituyéndola por programas académicos que más parecen intentos por recaudar fondos.

Aquellos que, en un acto de valentía y cierta cínica dignidad deciden embarcarse en la aventura de "la tesis" deben afrontar un mar de trabas burocráticas y de exagerado perfeccionismo en caso de caer en manos de revisores inadecuados. Parece que no se entiende que la elaboración de una tesis es, en muchos casos, un primer salto a la piscina de investigación, un proceso de aprendizaje.

Las instituciones de investigación, en muchos casos, carecen de recursos suficientes para una adecuada interacción con los alumnos durante la formación médica, limitando el acceso de los estudiantes a los verdaderos expertos y potenciales mentores hasta una etapa muy posterior en su desarrollo. En este sentido, pensamos que mejorar la comunicación entre aquellos que realizan investigación como su principal actividad y aquellos que se encuentran en formación es un paso vital para aprovechar el conocimiento alcanzado y potenciarlo. Esto debe ir de la mano con hacer flexible la currícula en la cual se deje espacio para este tipo de crecimiento personal. 